\title{
Acquisition and extinction of differential responses to signals paired with shock or shock omission in goldfish: Evidence for truly discriminated avoidance learning
}

\author{
DOMINIC J. ZERBOLIO, JR., and JOEL L. ROYALTY \\ University of Missouri, St. Louis, Missouri
}

\begin{abstract}
Goldfish trained to discriminate between signals paired with shock (S-) and signals paired with shock omission (S+) with a linear presentation procedure, originally learned (OL) to control the signal state of a shuttle box and showed a decided preference for the S+ signal. In Experiment 1, following OL, groups had one OL signal replaced (S+ or S-), both signals replaced (S+ and S-), or the OL signals reversed (S+ and S- reversed) and were then tested in a transfer training procedure. In transfer, groups with one signal replaced maintained discriminated performance at OL levels; the S+ replaced group was slightly superior to the S- replaced group on the first day of transfer. With both OL signals replaced, discrimination dropped to chance performance levels, whereas, with OL signal shock pairing reversed, discrimination performance dropped below chance levels. In Experiment 2, following OL, extinction procedures consisted of turning off the shocker (0\% shock) or of shocking $100 \%$ or a random $25 \%$ of the trials. A fourth extinction procedure $\left(R_{1}\right)$ retained the trial start response-dependent shock-omission contingency, but shock differentiating the S+ and S- signals was eliminated entirely. Extinction of the $\mathbf{S}+/ \mathrm{S}-$ discrimination was measured both during extinction training per se and with reversal retraining of the $\mathbf{S}+/ \mathbf{S}-$ discrimination later. Groups for which the $\mathrm{OL} \mathbf{S}+$ was paired with shock during extinction extinguished on both measures, but groups for which the OL Swas paired with shock omission did not extinguish, especially as shown by the reversal test procedure. Theoretical implications and the implications for several conditioning procedures are discussed.
\end{abstract}

Discrimination learning, in which the animal learns to respond differentially to two different stimuli, has long been the all but exclusive province of researchers using positive reinforcement procedures for the simple reason that animals learn a discriminated response easily when the differential signal-specific reinforcement is food/food-omission but very slowly, if at all, when the signal-specific reinforcement is shock/shockomission.

While instances of discrimination learning using shock/shock-omission procedures have been reported (e.g., Jacobs, 1963; Zerbolio \& Reynierse, 1967), they are rare and have not dispelled the notion that discrimination learning appears to be a function of the kind of reinforcer applied.

This discrepancy may be understood by considering the typical discrimination learning task in which two signals are simultaneously presented in a T- or Ymaze. If the reinforcement procedure entails food/ food-omission, the animal is food deprived and, consequently, fairly active. However, even if it does not move on the first trial, it is typically prodded out of

The authors' mailing address is: Department of Psychology, University of Missouri, St. Louis, Missouri 63121. the start alley. In either case, it automatically "chooses" one of the alternative alleys and has a $50 \%$ chance of finding food in the alley that it enters. After a few trials, the probability is high that it will have found food in one of the alleys and that it will leave the start alley of its own accord. Thus, food deprivation all but guarantees that the animal will respond on a large number of trials, so the experimenter can provide differential reinforcement.

Now consider the negative reinforcement analog of this procedure in which food/food-omission is replaced by differential shock/shock-omission. Again, on the first few trials, the animal may respond and be shocked $50 \%$ of the time. If, however, the animal fails to respond, the experimenter shocks it to force it to move from the start alley. The first few trials, then, are characterized by shock following some responses and shock following failures to respond.

While it is true that even for the randomly responding (nondiscriminating) animal there is a differential shock probability associated with responding vs. not responding, an awareness of this differential probability can only be established via continuing activity on the part of the animal. Unfortunately, the per- 
vasiveness of shock on early trials results not in continued activity but, rather, in a species-specific defense reaction (Bolles, 1970) to shock-freezing. Once this freezing pattern develops, the animal rarely leaves the start alley before being blasted out by shock, and the reinforcement procedure deteriorates into a differential shock-escape procedure. Thus, contrary to the food/food-omission procedure, the shock/shockomission procedure all but guarantees that the animal will cease responding and that the experimenter will have no opportunity to provide differential reinforcement for choice behavior.

For the animal with a high propensity to freeze in procedures involving shock, the wonder is not that it learns a discriminated response slowly or rarely but, rather, that it ever learns it at all. Fortunately, not all animals have a high propensity to freeze in situations involving shock. Goldfish continue to respond before shock even in situations in which they cannot control it (Woodard \& Bitterman, 1973; Zerbolio \& Wickstra, 1978a, 1978b). Because goldfish fail to freeze, they would be expected to provide the experimenter with the opportunity to differentially reinforce signal-specific responses with shock or shockomission reinforcement.

Zerbolio and Wickstra $(1979,1980)$, using a simultaneous presentation procedure in a Y-maze, showed that goldfish could learn to leave the start (TS signal) alley and swim to the alternative alley, which contained a signal paired with shock omission. This response accomplished two things. It changed the goldfish's physical position in the maze and changed the signal condition of its immediate surrounds.

Because the shuttlebox, unlike the Y-maze, cannot be used to simultaneously provide the animal with a choice between shock-paired and shock-omissionpaired signals subsequent to its response to the TS signal, a new discrimination technique was developed to accomplish, in the shuttlebox, the same signalchange result that is obtained in the Y-maze.

This technique, the linear presentation procedure, utilizes response-contingent signal changes in the shuttlebox. As in the Y-maze, there are three signals. Trial start is indicated by a TS signal. The goldfish's first response $\left(R_{1}\right)$ randomly changes the signal state of the entire shuttlebox to one of the other two signals, which are differentially paired with shock (S-) and shock omission $(\mathrm{S}+)$. Subsequent responses alternate the signal state between $\mathrm{S}+$ and $\mathrm{S}-$. Shock is programmed to occur $10 \mathrm{sec}$ after trial onset $\left(T_{10}\right)$, and its delivery or omission is determined by the signal state that prevails at $T_{10}$. If the signal state at $T_{10}$ is either the TS signal (indicating no response) or $\mathrm{S}-$, shock is delivered. If the signal state is $\mathbf{S}+$, shock is omitted.

Using the linear presentation procedure, Zerbolio (1981) showed that goldfish learn to control the sig- nal state of the shuttlebox and show a decided preference for the $\mathrm{S}+$ signal state. It was also found that reversal of signal-shock pairing produced reversal of the signal preference. The linear presentation procedure, then, represents a technique that can be applied to both the investigation of discrimination per se and the examination of avoidance learning. Because very little is known about either discrimination or avoidance learning generated by this procedure, the aim of the present experiments was to further examine the specifics of discrimination and avoidance that have been made accessible by this technique.

\section{EXPERIMENT 1}

Jenkins (1965), among others, pointed out that an animal that learns only either to approach $S+$ or to avoid $S$ - could perform a simultaneous presentation discrimination task as well as an animal using both signals. Most research, however, has shown that animals do use both signals (Mackintosh, 1974). The use of both signals, however, need not imply that both are equally important in controlling behavior, although Derdzinski and Warren (1969) found that this was indeed the case for cats faced with a food/ food-omission task.

On the other hand, Mason, Stevens, Wixon, and Owens (1980) and Stevens, Mason, and Wixon (1981) reported that, for rats faced with the same task, the S - signal appears to be more important. Mandler (1968) found that the $S$ - signal was more important for rats just after learning a food/food-omission task, but that the $\mathrm{S}+$ signal became dominant with overtraining. Stevens and Fechter (1968) also found that the S- signal was more important for rats when the reinforcer was water, but, in a shock-escape task, the $\mathrm{S}+$ signal was more important.

Determining the relative importance of the $\mathrm{S}+$ and $\mathrm{S}-$ signals in discrimination learning is fairly easy. Following original learning $(\mathrm{OL})$ of the discrimination, training continues on a transfer task in which one or both of the OL signals are replaced with new and presumably neutral signals. If the $S-$ signal is more important, animals with the OL S- signal replaced should show a greater degree of disruption (negative transfer) than animals with the OL S+ signal replaced. If the $S+$ signal is more important, the opposite result should occur. If the $\mathrm{S}+$ and $\mathrm{S}-$ signals are of equal importance, animals with $\mathrm{S}+$ replaced should perform equivalently to those with $\mathrm{S}-$ replaced.

However, equivalent performance with either the $\mathrm{S}+$ or $\mathrm{S}-$ signal replaced says nothing about the actual level of discrimination. Obviously, "good" performance in transfer means discrimination levels at or near those reached at the end of OL. But what is "poor" discrimination performance? If the animal, 
in transfer, cannot discriminate between the $\mathrm{S}+$ and $\mathrm{S}$ - signals, it would be expected to choose randomly between them. If both OL signals were replaced in transfer, the animal could not know, initially, which signal was the $S+$ and which was the $S-$, and its discrimination performance would be expected to drop to chance levels. Thus, animals with both signals replaced would establish the baseline for "poor" or no discrimination, just as the level of discrimination at the end of OL establishes the baseline for "good" discrimination (Zerbolio, 1981). Since animals with both signals replaced would also be expected to learn to discriminate between their new (replaced) $\mathrm{S}+$ and $S-$ signals with continued transfer training, they would also establish the baseline rate of learning the $\mathrm{S}+$ and $\mathrm{S}-$ properties of the new signals.

The consideration of the actual levels of discrimination within the parameters of the upper and lower control baselines has strong implications regarding the absolute as well as the relative importance of $\mathrm{S}+$ and $\mathrm{S}$ - signals. For example, an animal that learned to discriminate on the basis of only one signal in OL, as suggested by Jenkins (1965), should discriminate at or near the level reached at the end of OL if the critical signal were retained in transfer. If its learned signal were replaced in transfer, it should discriminate at a level equivalent to that of animals for which both signals were replaced in transfer.

Experiment 1 was designed to assess, via signal replacement in transfer, the specific discrimination control characteristics of the OL S+ and S- signals acquired with the linear presentation training procedure. In addition to replacing one or both of the OL signals, a transfer condition in which the OL signals were reversed was included to further establish negative transfer discrimination baselines for comparative purposes. Based on Zerbolio's (1981) results, reversing the OL pairing in transfer was expected to produce maximum disruption-a condition characterized by discrimination well below chance levels during the initial stage of transfer learning.

\section{Method}

Subjects. Sixty-four 5-6-cm-long goldfish, obtained from Ozark Fisheries and housed in individual $7.5 \times 11.5 \times 12.5 \mathrm{~cm}$ deep aquaria, served as subjects. Housing was well filtered, with both temperature $\left(21.1^{\circ} \mathrm{C}\right)$ and $\mathrm{pH}(7 \pm .1)$ held constant throughout the experimental manipulation. The animals were fed daily. Approximately one-third of the water was siphoned out and replaced daily; the water was completely changed weekly. Siphoning served to remove any debris that was not removed by the filtering system.

Apparatus. Four identical $29.2 \times 11.4 \times 11.4 \mathrm{~cm}$ deep shuttletanks, designed for aquatic species and similar to those described by Horner, Longo, and Bitterman (1961) were used. They contained a center hurdle, $6.35 \mathrm{~cm}$ high, and had $45-\mathrm{deg}$ sloping sides and a 9-cm-long flat top. Water clearance was $2 \mathrm{~cm}$ over the hurdle top at all times. Shuttling activity was monitored by photocells focused across the top of the hurdle, at the ends of the top flat portion, $9 \mathrm{~cm}$ apart. Photocell light sources were $2.5-\mathrm{V}$ dc prefocused penlight bulbs run at $1.5-\mathrm{V}$ ac to increase bulb life. Sig- nal color cues were provided by $7-\mathrm{W} 110-\mathrm{V}$ ac Christmas tree lamps. The colors were red, yellow, green, and blue, with measured spectral peaks at $620 \pm 4,586 \pm 2,517 \pm 2$, and $474 \pm 2 \mu$, respectively. In addition, a clear $7-\mathrm{W} 110-\mathrm{V}$ ac lamp with a $650-\mathbf{9}$ 20-W resistor in series to reduce brightness served to illuminate the intertrial interval period (ITI). Shock, at $7 \mathrm{~V}$ ac $(.69 \mathrm{~V} / \mathrm{cm})$ in 200 -msec pulses, was delivered via $28 \times 10.2 \mathrm{~cm} 22$-gauge stainless steel plates which lined the interior sides of each tank. All events were programmed and recorded via appropriate circuitry. Approximately one-third of the water was siphoned from each shuttletank daily, which served both to replace water and to clean up any debris. Shuttletanks were aerated constantly.

Procedure. All goldfish were run 40 trials a day for 20 days with a variable ITI of $60 \mathrm{sec}$. The experiment was divided into two phases, 15 days of original learning (OL), in which all animals were treated alike, and 5 days of transfer learning (TL). The linear presentation procedure (Zerbolio, 1981) was used throughout both phases. With this procedure, a trial is a timed period with two 200-msec shock pulses scheduled to occur $10\left(T_{10}\right)$ and $11\left(T_{11}\right) \sec$ after trial onset. The ITI was signaled by clear lamps. To start a trial, all illumination was extinguished, which served as the trial start (TS) signal. A response was defined as the animal's swimming from one end of the shuttlebox, across the center hurdle, and through the photocell detector to the other end. The first response $\left(R_{1}\right)$ caused the termination of the TS signal and the onset, at random (Gellerman series), of either a shock-paired signal (S-) or a signal paired with shock omission $(\mathrm{S}+)$. Subsequent responses caused alternation of the $S+$ and $S-$ signals (i.e., from $S+$ to $S-$ or from $S-$ to $S+$ ). Thus, the goldfish could control the signal state (TS, $\mathrm{S}_{+}$, or $\mathrm{S}_{-}$) of the shuttlebox with its response via the response-contingent signal change programming. Shock omission was contingent on the signal state of the shuttlebox at $T_{10}$ and $T_{11}$. If the TS signal (indicating no response on the trial) or $S$ - signal prevailed at $T_{10}$ or $T_{11}$, shock was delivered as scheduled. If, however, the $S+$ signal prevailed during the $T_{10}$ to $T_{11}$ interval, shock was omitted. To discriminate successfully, the animal had to cause the $\mathrm{S}+$ signal to prevail during the entire shock delivery period, not just at $T_{10}$ or $T_{11}$. If $S+$ prevailed at $T_{10}$ and the goldfish shuttled in the 800 -msec intershock interval, the signal state alternated to $\mathrm{S}-$ and the shock at $\mathrm{T}_{11}$ was delivered as scheduled. If the $\mathrm{S}-$ signal prevailed at $T_{10}$, the first shock was delivered as scheduled. If the goldfish shuttled in the 800 -msec intershock interval, the signal state changed to $S+$ and the second shock at $T_{11}$ was omitted. If the goldfish did not shuttle prior to $T_{10}$, the prevailing signal state was TS and the first shock was delivered. If the animal then responded to the first shock, half the time the $S+$ signal would occur and half the time the S- signal would occur. If the S- signal occurred, shock was delivered, but if the S+ signal occurred, the second shock at $T_{11}$ was omitted. Shock at $T_{10}$ and $T_{11}$ was entirely dependent upon the prevailing signal state of the shuttlebox at the scheduled times of delivery, and the signal state was entirely dependent on the goldfish's shuttling responses. An avoidance or discriminated response was recorded only if both shocks were omitted. The trial period was a total of $11.2 \mathrm{seclong}$, and the ITI signal condition (clear bulb illumination) was reestablished at the end of each trial.

For the first 15 days of training, all animals were treated alike except for the color signals serving as $S+$ and $S-$. Groups of 16 animals had green $\mathbf{S}+$ /blue $\mathbf{S}-$, yellow $\mathrm{S}+$ /red $\mathbf{S}-$, blue $\mathrm{S}+$ / green $\mathbf{S}-$, and red $\mathrm{S}+$ /yellow $\mathrm{S}-$. Thus, color and shock pairing were completely balanced in $\mathrm{OL}$.

In the transfer phase, on Days 16 to 20 , one group of 16 goldfish had both of its OL signals reversed (e.g., the OL green S+1 blue $S-$ became TL blue $S+$ /green $S-$ ), a second group of 16 had its OL S+ signal replaced (e.g., the OL blue S+/green Sbecame red $S+/ g r e e n ~ S-$ ), a third group of 16 had its OL S- signal replaced (e.g., the OL yellow $S+/$ red $S-$ became yellow $S+$ / blue $S-$ ), and the last group of 16 had both of its OL signals replaced (e.g., the OL green $S+/$ blue $S-$ became yellow $S+/$ red 
S-). Colors and shock pairing were balanced as far as possible in all groups.

Illumination signals were delivered via three lamps mounted at each end of the shuttlebox, with illumination diffused through translucent plastic plates. The center lamp at each end was the clear ITI lamp. The S+ and S- lamps were to the left and right of the ITI lamp. The S+ lamp was on the right at one end and on the left at the other end, so that it was not in the same relative position on both ends of the tank. This placement was necessary because earlier work in our lab had shown that goldfish could learn to respond to a lamp's position instead of to its color. Thus, position was balanced for all S+ and S- placement positions. Earlier, Zerbolio (1981) had used an ITI illumination composed of the simultaneously illuminated $\mathrm{S}+$ and $\mathrm{S}-$ signals. In that study, which involved both an original learning and a reversal learning phase, the ITI illumination did not change with phase because the same signal colors were used throughout. In the present work, if the ITI illumination were the composite of both the S+ and Ssignals, changing one or both of the color signals would have produced an ITI signal change as well. To eliminate this difference between OL and TL, ITI illumination was held constant by using clear lamps throughout.

Two measures were taken daily for each goldfish-the number of trials with response (TwR) and the number of trials in which the $\mathrm{S}+$ signal prevailed throughout the shock-delivery period $(\mathrm{S}+)$. The TwR measure indicates the number of trials in which, via its initial response, the goldfish gained access to the S+ and/or Ssignals. However, to perform successfully, the goldfish had to control the $\mathrm{S}+$ and $\mathrm{S}-$ signals following access so that the $\mathrm{S}+$ signal prevailed during the shock delivery period to produce the omission of the shock.

Note that the presence of the S+ signal on any given trial is not necessarily evidence of discrimination. Nor is a simple increase in the number of $S+$ trials an adequate measure of discrimination, since an S+ increase would occur with an increase in TwR. To differentiate between the randomly responding and the discriminating animal, a "discrimination index" (DI) was calculated for each animal on each day of training. The DI was defined as [(S+) $(S-)] / 40$, where 40 is the number of trials per day, $S+$ is the number of $S+$ trials per day, and $S-$ is the number of $S-$ trials per day. Note that, since $T w R=(S+)+(S-), S-=T w R-(S+)$. This ratio has limits of \pm 1.00 . A cursory examination of the DI reveals that it (1) differentiates between discriminated and random behavior, (2) reflects the requirement of an initial response, and (3) provides a measure of signal preference. Therefore, the randomly responding animal for which S+ and S- trials are equal will have a DI of zero. Since the denominator of the ratio is 40 rather than actual TwR, the DI distinguishes between the discrimination performance of animals that respond on just a few trials and those that respond on many trials per day. Finally, as an index of signal preference, the DI will be positive if the animal prefers the $\mathrm{S}+$ and negative if it prefers the $\mathrm{S}-$ signal. Thus, it provides a convenient measure of positive and negative transfer effects. Other measures, such as the ITI responses, were not found to be informative in the present or in earlier work (Zerbolio, 1981) and were therefore omitted.

\section{Results and Discussion}

Original learning. All animals learned to respond discriminatively during the 15 days of original learning, as shown in Figure 1. Specifically, goldfish learned to respond on increasingly more trials (TwR), learned to control the linearly presented signals so that $\mathrm{S}+$ prevailed throughout the shock delivery period on increasingly more trials, and learned to discriminate between the $\mathbf{S}+$ and $\mathrm{S}-$ signals with their responses.

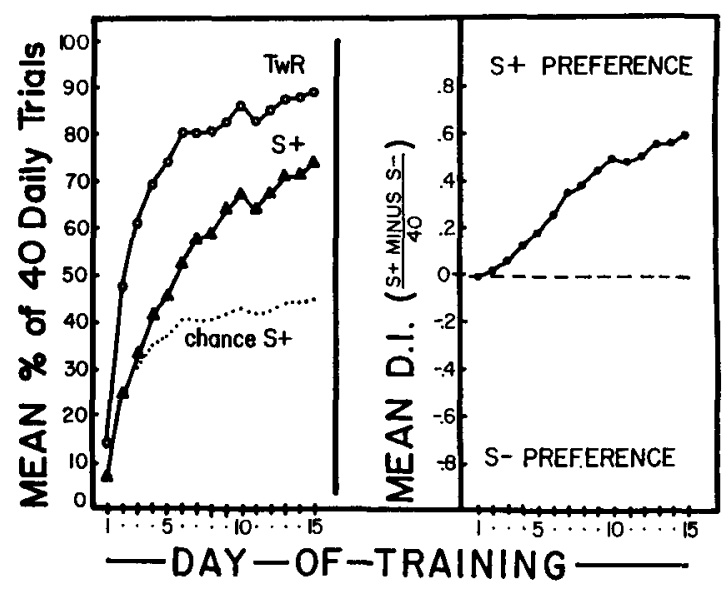

Figure 1. The trials with response (TwR) and $S+$ at $T_{10}$ measures appear in the left panel along with the chance $S+$ level $(.5 \times \mathrm{TwR})$. The discrimination index (DI) measure appears in the right panel for the 15 days of original learning in Experiuent 1.

These impressions were confirmed by one-way repeated ANOVAs of the OL data. For the TwR measure, a significant increase in TwR with training was observed $[F(14,882)=244.11, p<.01]$. A comparable increase was found for the $S+$ measure $[F(14$, $882)=221.07, p<.01]$. Both the TwR and $S+$ measures, as a percentage of the 40 daily trials, appear in the left panel of Figure 1. In addition, chance S+ levels, or half of the daily TwR rate (TwR $\times .5)$, are shown for comparison purposes. The discrimination index (DI) for OL showed a significant increase in S+ preference during OL training $[F(14,882)=123.72$, $\mathrm{p}<.01$ ]. The DI measure, over days of OL training, appears in the right panel of Figure 1.

It is clear that during OL, all animals learned to discriminatively control the $\mathrm{S}+$ and $\mathrm{S}-$ signals and, thus, to avoid shock by making the $S+$ signal prevail throughout the shock delivery period on a substantial majority of the trials.

Transfer training. The results of the transfer training task are shown in Figure 2. For the TwR measure, no differences between groups, for transfer training, or their interaction were found. The combined mean daily TwR as a percentage of the daily trials is shown in the left panel of Figure 2. In addition, the TwR for the last day of OL and the chance $\mathrm{S}+$ expectancy $(\mathrm{TwR} \times .5)$ are shown. From these data, it is clear that replacing or rearranging the $\mathrm{OL}$ signals did not affect the number of trials with response.

A substantially different picture emerged with the $\mathrm{S}+$ and DI measures. First, note that the groups did not differ on either the $\mathrm{S}+$ or DI measures on the last day of OL training. For the reversal group, the 1st day of transfer training produced a substantial drop from its $\mathrm{OL}$ discrimination level $[\mathrm{t}(15)=17.62, \mathrm{p}<.01]$ 


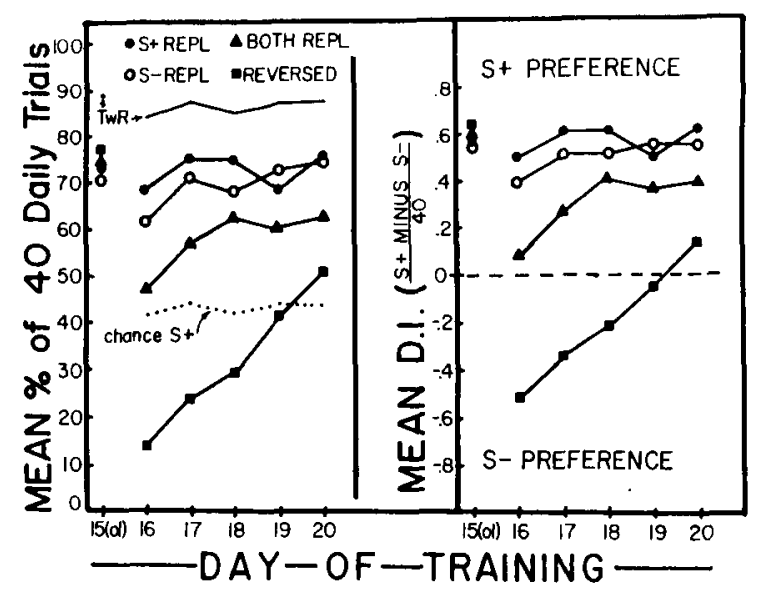

Figure 2. The performance of groups with the S+ signal, Ssignal, or both signals replaced, and the group with the OL signalshock pairing reversed for the 5 days of transfer training are shown. The left panel shows the $T w R$ (combined), chance $S+(.5 \times T w R)$, and the $S+$ performances for each transfer group, with their last OL day performance for comparison purposes. The right panel shows the DI measure for each transfer group, including the last OL day for each.

and a drop well below chance discrimination levels $[t(15)=12.63, p<.01]$. Since the $S+$ and DI measures provided redundant information in the first experiment, only the DI statistical analysis results are reported. It is obvious that the reversal group continued to prefer the OL S+ signal during transfer, which is, of course, the transfer shock-paired signal. It required 5 days of transfer training for the reversal group to acquire a preference for the transfer $\mathrm{S}+$ signal over the transfer $S-$ signal, and even then the preference is not statistically reliable $[t(15)=1.62]$. However, it is clear that, with continued training, the reversal group would learn to prefer the reversed S+ signal, a result shown earlier by Zerbolio (1981) for a similar task.

The group with both signals replaced with new and presumably neutral signals in transfer would not be expected to know which new signal was paired with shock or shock omission, and therefore would be expected to show a substantial drop from its OL discrimination performance to chance expectancy levels on the 1st day of transfer. On the 1st day of transfer, the group with both signals replaced showed this substantial drop from the previous OL training day $[t(15)=6.60, p<.01]$ and did not differ from a chance discrimination [ $t(15)=1.37]$. However, by the 2nd day of transfer training, the both-signals-replaced group showed $\mathrm{S}+$ preference above chance expectation levels $[\mathrm{t}(15)=4.10, \mathrm{p}<.01]$.

The last two groups, with only one of their OL signals replaced in the transfer training phase, are the most interesting. In transfer, the group with its OL $S+$ signal replaced (and OL $S$ - signal retained) and the group with its OL S- signal replaced (and OL $\mathrm{S}+$ signal retained) did not differ in discrimination performance. However, on the 1st day of transfer, when each was compared with its own last OL day discrimination performance, the OL S+ signal-replaced group did not differ from its OL discrimination level $[\mathrm{t}(15)=1.04]$, whereas the group with its OL S- signal replaced showed a small, but significant, drop from its OL discrimination level $[t(15)=3.63, p<.01]$. Both groups continued to discriminate well above chance levels $[S+$ replaced, $t(15)=15.52, p<.01$; $S-$ replaced, $t(15)=9.46, p<.01]$.

\section{Discussion}

These data are problematic for a traditional twofactor position. The two-factor position assumes that avoidance occurs because the shock-paired signal comes to elicit fear and the animal learns to escape from the fear-eliciting signal. As long as the shockpaired signal is retained in transfer, avoidance would be expected to continue to occur. Since, in this case, the avoidance response is the discriminated response, the animal would also be expected to continue to discriminate.

But the group with the OL S- signal replaced, in transfer, sees the OL S+ signal and a new signal that replaces the OL S- signal. According to the traditional two-factor position (Mowrer, 1947), it should not know which signal to avoid since, prior to transfer training, neither signal has been paired with shock. As a result, its discrimination performance should be disrupted, at least in the early part of transfer training. Note that the group with the OL S- signal replaced shows very little disruption.

One could argue that the S- replaced group learned very rapidly that the new signal (the $S$ - replacement) was paired with shock and thus maintained its discrimination performance. But this explanation makes at least two assumptions. The first is that "very rapid learning" about the shock pairing of the $S$ - replacement signal occurred early on the 1st day of transfer training. The second is that the S+ signal was of very little importance. But this same set of assumptions ought to hold for the group with both OL signals replaced.

If the importance of the $\mathrm{S}+$ signal is assumed to be negligible, then the both-signals-replaced and S--signal-replaced groups logically have identical opportunities to learn the shock-paired property of the new (replaced) S- signal. With the same opportunity to learn, both groups would be expected to perform equivalently; that is, the both-signals-replaced group should learn about the new S- signal just as fast as the S--signal-replaced group.

However, as these data show, on the first day of transfer training, the S--replaced group discriminated significantly better than the both-signals- 
replaced group [ $\mathrm{t}(30)=3.92, \mathrm{p}<.01]$. Furthermore, earlier analyses showed that the $\mathrm{S}$--replaced group maintained discrimination performance well above chance levels, whereas the both-signals-replaced group dropped to chance discrimination performance on the 1st day of transfer. These findings, argue against any sort of rapid learning hypothesis.

However, one must still account for the continued discrimination performance of the S--replaced group in transfer. The most reasonable way to do this is to assume that the remaining $\mathrm{S}+$ signal is important. This assumption also explains why the both-signalsreplaced group's discrimination level dropped to chance on the 1st day of transfer.

With both signals replaced, the goldfish has no old information to use and must learn everything anew. With the S- signal replaced, the goldfish has the OL $\mathrm{S}+$ signal retained, and, as long as it responds to maintain the $\mathrm{S}+$ signal, it discriminates.

Clearly, these data support the notion that goldfish learn the motivational properties of both the $\mathrm{S}+$ and $\mathrm{S}$ - signals in OL. Furthermore, as long as one OL signal is retained in transfer, the goldfish uses the retained signal and the associated information to maintain its discriminated performance. In addition, there seems to be a slight superiority of the Ssignal in that when the S+-replaced and S--replaced groups are compared with their own discriminated performance immediately before transfer training, the $\mathrm{S}+$-replaced group (which retained the $\mathrm{S}-$ signal) does not differ from its pretransfer level, whereas the $\mathrm{S}$--replaced group (which retained the $\mathrm{S}+$ signal) shows a slight, but significant, drop in discrimination performance, although only on the 1st day of transfer.

As a whole, the present data are quite consistent with the results of discrimination learning studies using food/food-omission reinforcement techniques (e.g., Stevens, Mason, \& Wixon, 1981) in that, not only do the animals learn to discriminate between the differentially reinforced signal conditions, but also the $\mathrm{S}-$ signal is more important than the $\mathrm{S}+$ signal when importance of specific signals is determined using signal-replacement transfer procedures. The similarity is even more apparent when one considers the meaning of the $\mathrm{S}+$ and $\mathrm{S}-$ signals. If one allows the sign (+ or - ) to represent the state the animal prefers rather than the signal associated with the application of the physical reinforcer, it becomes obvious that animals prefer signals associated with states of food presentation or shock omission and do not prefer signals associated with states of food omission or shock. Determination of preferred state is simple enough. All one has to do is give the animal the opportunity to choose. The animal will learn to approach the signal associated with the preferred state and to avoid the signal associated with the nonpreferred state.
With the present and earlier discrimination work in mind, then, it is obvious that animals will learn to approach signals associated with preferred states ( $\mathrm{S}+$ : shock omission or food) and to avoid signals associated with nonpreferred states ( $\mathrm{S}-$ : food omission or shock). It seems reasonable to allow the animal to operationally define the sign (preference) via its approach or avoidance responses rather than to adhere to some other sort of arbitrary convention in which sign is determined for the convenience of the experimenter and independently of the animal's response (and preference).

Viewed as an example of discrimination learning, there is nothing particularly surprising about the results of Experiment 1 until one recalls that the reinforcer is the differential application of shock/shockomission and the discriminated response is therefore, by definition, an avoidance response.

But the discrepancy in frequency of demonstrations of discrimination learning based on food/foodomission and shock/shock-omission is not the only difference in the literature for these different reinforcement procedures. Another difference is in the resistance to extinction between food-reinforced responses and shock-avoidance responses. Typically, when comparable tasks and response measures are involved, an avoidance response is more resistant to extinction than is a comparable food-reinforced response. The difference in resistance to extinction is typically a difference in the number of trials with response (TwR) after the institution of extinction procedures. It is quite possible that the TwR measure is an inadequate measure of extinction for the goldfish. If so, the linear presentation procedure, with its additional $\mathrm{S}+/ \mathrm{S}-$ discrimination learning measure, the DI, and its transfer design capability, may help to clarify the extinction process for this animal. Experiment 2 was designed to explore this possibility.

\section{EXPERIMENT 2}

Much of the controversy surrounding the study of avoidance learning in goldfish is a result of the failure to appreciate the implications of applying traditional activity rate measures of avoidance learning to an animal which demonstrates an increase in activity both in the presence and in the absence of shock, does not habituate by ceasing to respond, and does not freeze in procedures using shock (Steiner, 1971; Zerbolio, 1981).

These implications also extend to the measurement of extinction. In an examination of avoidance extinction in goldfish, Wallace and Scobie (1977) found that goldfish behave very much like other laboratory animals except for one seemingly anomalous result. When the extinction procedure consisted of responseindependent shock on every trial, goldfish main- 
tained their TwR rates at levels near those of control animals for which the avoidance contingency remained in effect. Since the TwR rate was the learning measure, it appeared as if this experimental extinction procedure did not produce extinction of the avoidance response.

Wallace and Scobie's $100 \%$ shock-extinction procedure was applied in recognition of the controversy about what constitutes the appropriate paradigm to extinguish an avoidance response. This issue was triggered by Davenport and Olson (1968), who argued that, since the typical procedure of disconnecting the shocker did nothing to disconfirm the animal's expectancy that a response would be followed by shock omission, it was not reasonable to expect the response to extinguish.

The issue of extinction as the disconfirmation of expectancies acquired in original learning can be crystallized by considering what happens in a foodreinforced procedure. Following acquisition, in which the animal learns to expect food following its response, the food dispenser is disconnected. In extinction, when the animal finds no food following its response, its expectancy about the relationship between responding and food is disconfirmed and the animal stops responding.

For the avoiding animal, the procedure of disconnecting the shocker does not disconfirm the OLacquired expectancy of response/shock-omission because responses continue to be followed by shock omission. As Mackintosh (1974) observed, the wonder is not that the avoidance-trained animal is slow to extinguish but, rather, that it ever extinguishes at all.

If experimental extinction is defined as a procedure which disconfirms the response-reinforcement expectancy acquired in OL, the avoidance analog of the extinction of positively reinforced behavior is to shock the animal on every trial, or to shock it on a random number of trials, independent of its response. Both procedures substantially reduce the resistance to extinction (Bolles, Moot, \& Grossen, 1971; Davenport \& Olson, 1968).

The aim of Experiment 2 was to examine the extinction of an avoidance response in goldfish when the avoidance response was the discriminated avoidance response produced by the linear presentation procedure. In addition to the traditional extinction procedure of disconnecting the shocker, other procedures designed to extinguish the $\mathrm{S}+/ \mathrm{S}-$ differential expectancies acquired in original learning were included.

As a discrimination-learning paradigm, the linear discrimination procedure offers the additional advantage of being amenable to the application of transfer procedures to measure the strength of responses associated with specific signals. That is, if extinction procedures actually do extinguish originally learned signal-specific responses, later reversal of the shock and shock-omission pairing of the signals should not affect the reacquisition of a discrimination between these signals. If, on the other hand, extinction procedures do not extinguish the OL responses, reversal of the originally learned signalspecific shock pairing should disrupt the reacquisition of the discrimination.

\section{Method}

Subjects. Forty-eight additional 5-6-cm-long goldfish, obtained and maintained as in Experiment 1, were used. The goldfish were received from the shipper with salmonella and were treated prophylactically prior to experimental use in order to ensure their health for the 40-day period of the experiment. The details of the prophylactic treatment are available from the first author upon request. Except for the prophylactic treatment, the goldfish were treated identically to those in Experiment 1.

Apparatus. The apparatus was the same as that used in Experiment 1 except that only the red and green lamps were used.

Procedure. Experiment 2 was divided into the following four phases of 10 training-test days each: original learning (OL), extinction, TwR retraining, and reversal testing. The linear presentation procedure or some variant (i.e., extinction and TwR retraining) of it was used throughout. In all phases, the colors serving as $\mathrm{S}+$ and $\mathrm{S}-$ were balanced in all groups.

Using the linear presentation procedure during the first 10 days, all animals were trained to discriminate between the $S+$ and $S-$ signals presented. As in Experiment 1, all animals were run $\mathbf{4 0}$ trials a day with a variable ITI of $60 \mathrm{sec}$. The ITI was the illumination of both the green and red lamps on both ends of the shuttletank (Zerbolio, 1981). Trial onset was indicated by dark, or by extinguishing both lamps. The animal's first response $\left(\mathbf{R}_{\mathbf{1}}\right)$ turned on, at random, the $S+$ or $S-$ signal (Gellerman series), and subsequent responses caused the alternation of the $S+$ and $S-$ signals. Shock, a single $200-\mathrm{msec} 7-\mathrm{V}$ ac pulse, was programmed for delivery at $10 \mathrm{sec}$ after trial onset $\left(T_{20}\right)$. During original learning, shock was omitted if the $S+$ signal prevailed at $T_{10}$ but delivered if the prevailing signal was the dark trial-start signal (indicating no response on that trial) or $\mathrm{S}-$. The trial was terminated following the shock delivery period at $10.2 \mathrm{sec}$ after trial onset and the ITI signal condition reestablished. All other conditions were identical to those of Experiment 1.

Following the first 10 days of $\mathrm{OL}$, the $\mathbf{4 8}$ goldfish were split into four groups of 12 goldfish each and run an additional 10 days, with each group receiving a different form of extinction training. These are detailed as follows:

For 0\% shock, the shock generator was disconnected. This is the traditional extinction procedure. Effectively, all signals, TS, $S-$, and $S+$, were unpaired. Response-contingent signal changes remained as in OL.

For $100 \%$ shock, shock was delivered at $T_{10}$ independent of the goldfish's response. Effectively, TS, S-, and S+ were equally paired with shock. Response-contingent signal changes remained as in OL.

For $R_{1}$, shock was delivered only if the goldfish failed to respond on a trial. If the goldfish responded at all, regardless of which signal $\left(S-\right.$ or $S+$ ) was present at $T_{10}$, the shock was omitted. Effectively, the TS signal remained paired with shock and, as in OL, the $S+$ signal was unpaired; but the $S-$ signal was also unpaired. Response-contingent signal changes remained as in the OL.

For $25 \%$ random $(25 \% \mathrm{R})$ shock, shock was delivered at $\mathrm{T}_{10}$ on a random $25 \%$ of all trials, independent of the goldfish's response. Effectively, each signal could be paired with shock $25 \%$ of the time and be unpaired $75 \%$ of the time. The $25 \%$ shock value was approximately comparable to the percentage of trials with shock 
on the last days of OL. Response-contingent signal changes remained as in OL.

At the end of extinction testing, the four groups differed markedly in the number of trials with response (TwR). Since the next phase planned was the reversal learning testing phase, and since the expected effect of the reversal procedure was anticipated to occur very early in reversal training, equating the four extinction groups on the TwR measure seemed propitious. The extinction data showed that this could be accomplished without affecting the discrimination level, other than producing additional extinction, by running all groups like the $\mathbf{R}_{1}$ extinction training group. Thus, all groups were run for an additional 10 days (Days 21 to 30 ) under procedures identical to the $R_{1}$ extinction testing conditions.

Finally, each of the extinction groups (0\% shock, $100 \%$ shock, $R_{1}$, and $25 \% R$ shock) were split into two halves of six goldfish each. Colors serving as $\mathrm{S}+$ and $\mathrm{S}-$ were balanced in each half. One half was retrained with its $\mathrm{S}+$ and $\mathrm{S}-$ signals paired with shock exactly as had occurred in its OL phase, and the remaining half of each group had its OL signal-shock pairing reversed. Each half was then retrained for an additional 10 days (Days 31 to 40) with the linear presentation procedure exactly as in OL, except for the reversed shock pairing for half of each extinction group.

\section{Results}

The TwR results for both the 10 days of $O L$ and the following 10 days of extinction for each extinction group ( $0 \%$ shock, $100 \%$ shock, $\left.R_{1}, 25 \% R\right)$ are shown in Figure 3.

Unlike the single-stimulus, discrete-trial avoidance paradigm in which TwR is the measure of avoidance, in the linear presentation procedure it is a measure which is only correlated with avoidance in the sense that it is a necessary prerequisite for discriminated avoidance. The TwR measure represents, however, a means of comparing the present results with those obtained under traditional avoidance procedures.

As can be seen in Figure 3, while all groups showed comparable and significant increases in TwR in OL $[F(9,396)=282.89, p<.01]$, a different picture emerged in extinction. The overall comparison showed

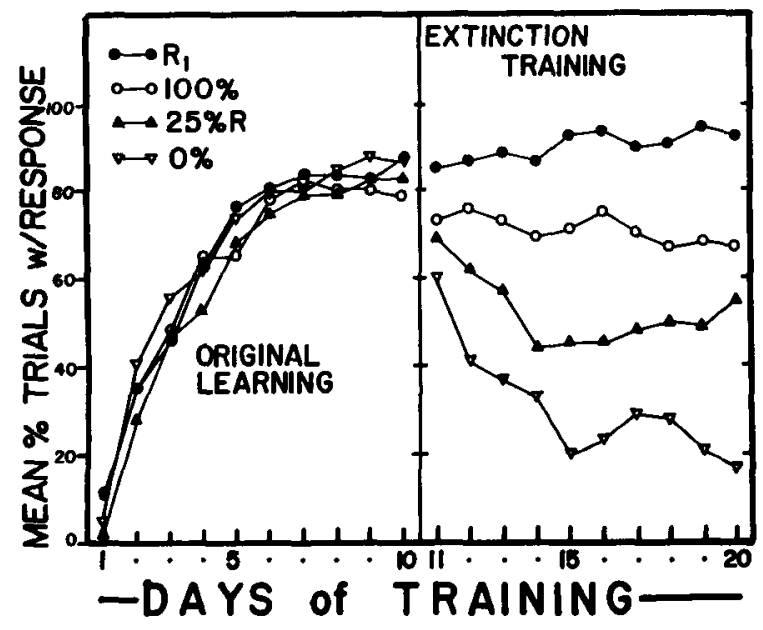

Figure 3. The original learning and extinction performances of the $0 \%, 100 \%, 25 \% R$ shock, and $R_{1}$ extinction groups on the trials with response (TwR) measure.

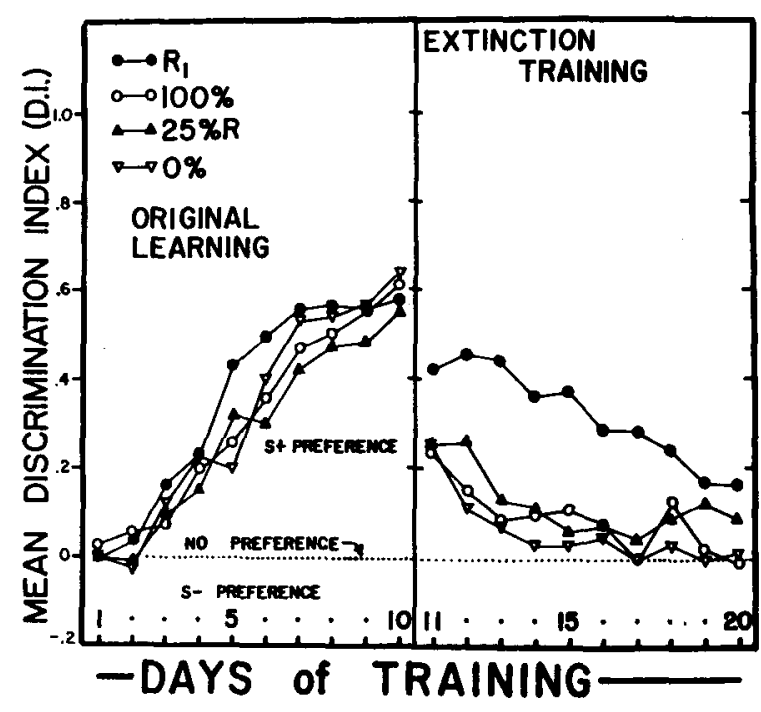

Figure 4. The original learning and extinction performances of the $0 \%, 100 \%, 25 \% \mathbf{R}$ shock, and $\mathbf{R}_{1}$ extinction groups on the discrimination index (DI) measure.

differences between groups $[F(3,44)=54.38, p<.01]$, days of training $[F(9,396)=7.09, p<.01]$, and a significant interaction $[F(27,396)=4.10, p<.01]$. Partitions of the main analysis showed that although the $100 \%$ shock extinction group responded on fewer trials than did the $\mathbf{R}_{1}$ group, neither changed their TwR rates over the 10 days of extinction ( $F s=.85$, .75 ). In contrast, both the $25 \% \mathrm{R}$ and $0 \%$ shock groups showed significant reductions in TwR in extinction $[12 \% \mathrm{R}, \mathrm{F}(9,396)=4.91, \mathrm{p}<.01 ; 0 \%$ shock, $F(9,396)=12.88, p<.01]$, with the $25 \% R$ group responding on more trials than the $0 \%$ shock group $[F(1,44)=20.08, p<.01]$ but on fewer than the $100 \%$ shock group $[F(1,44)=13.70, p<.01]$. Clearly, only the $25 \% \mathrm{R}$ and $0 \%$ shock extinction conditions produced a drop in the TwR measure.

The DI results for both $\mathrm{OL}$ and extinction are shown in Figure 4. All groups learned the discrimination equivalently during the 10 days of original learning. No group difference or training $\times$ group interaction was observed. It is also clear that while all groups apparently lost their OL discrimination on the DI measure, the $0 \%, 100 \%$, and $25 \% \mathrm{R}$ groups did so faster than the $\mathbf{R}_{\mathbf{1}}$ group. This was confirmed by the analysis of the extinction DI data, which showed overall significant differences among groups $[F(3,44)=$ $10.90, \mathrm{p}<.01]$, trial blocks $[\mathrm{F}(9,396)=17.65, \mathrm{p}<.01]$, and a significant interaction between groups and trials $[F(27,396)=1.89, p<.01]$. To understand the interaction, a second analysis of the DI extinction data with just $0 \%, 100 \%$, and $25 \% \mathrm{R}$ shock groups was performed. This analysis found only a significant drop in DI over extinction testing $[F(9,297)=12.69$, $\mathrm{p}<.01$ ]. No differences between groups or interactions were observed. Evidently, the $0 \%, 100 \%$, and 
$25 \% \mathrm{R}$ groups all lost the OL discrimination at the same rate. Additional partitions of the initial four groups analyses confirmed that the $\mathbf{R}_{\mathbf{1}}$ group did indeed drop on the DI measure $[F(9,396)=9.27, p<.01]$ and that although the $0 \%, 100 \%$, and $25 \% \mathrm{R}$ shock groups did not differ from each other, each differed from the $R_{1}$ group $[F s(1,44)=26.74,20.67,15.80$, respectively, all ps $<.01]$.

The effect of the reversal testing was anticipated to occur in the first few days of the reversal testing transfer procedure. Since the substantial differences between extinction groups on the TwR measure and the residual differences on the DI measure could mask the results of the reversal test, the groups were equated both on the TwR and DI measures by an additional 10 days (21 to 30 ) of extinction training with the $\mathbf{R}_{1}$ procedure.

The additional $R_{1}$ procedure equated all groups on the TwR measure and further extinguished any residual S+/S- discrimination such that, on Day 30 , all groups were responding on an average of $94 \%$ of their daily trials ( $\mathrm{TwR}=37.78$ ) and had an average DI of .056, which did not differ from zero discrimination $(t=1.59)$. Furthermore, neither groups nor halves of groups slated for OL vs. reversal testing later differed over the last 5 days (26 to 30 ) on either the TwR measure or the DI measure. It is clear that the additional $\mathbf{R}_{\mathbf{1}}$ extinction training served to stabilize and equate all groups at a high TwR level and to reduce any residual discrimination to zero or chance on the DI measure. The OL and reversal halves of each of the four extinction groups were analyzed separately on the DI measure. Figure 5 shows the average DI for the OL and reversal halves of each extinction condition as well as the performance of each half of each group on Day 30 for comparison purposes.

From Figure 5, it is clear that, in terms of reacquiring the $\mathrm{S}+/ \mathrm{S}-$ discrimination, the $\mathrm{OL}$ and reversed signal halves of the $100 \%$ and $25 \% \mathrm{R}$ groups did not differ, but that the OL halves of the $0 \%$ and $R_{1}$ groups both reacquired the $S+/ S-$ discrimination faster than their respective reversed-signal halves. Furthermore, the differences between halves of specific extinction groups were limited to the first 4 or 5 days of reversal training. These impressions were confirmed by statistical analysis. The reversal testing DI data were split into two segments covering Days 31 to 35 and Days 36 to 40 . No differences between groups or group halves and no significant interactions were observed over Days 36 to $\mathbf{4 0}$ on the DI measure, although all groups continued to improve their discrimination performance $[F(4,160)=7.04, p<.01]$. Thus, all differences due to the reversal procedure were confined to the first 5 days ( 31 to 35 ) of reversal testing, as expected.

During the first 5 days of reversal testing (Days 31 to 35 ), the OL and reversal halves of the $100 \%$ and $25 \% \mathrm{R}$ groups were not found to differ or to interact with training, but both groups improved their dis-

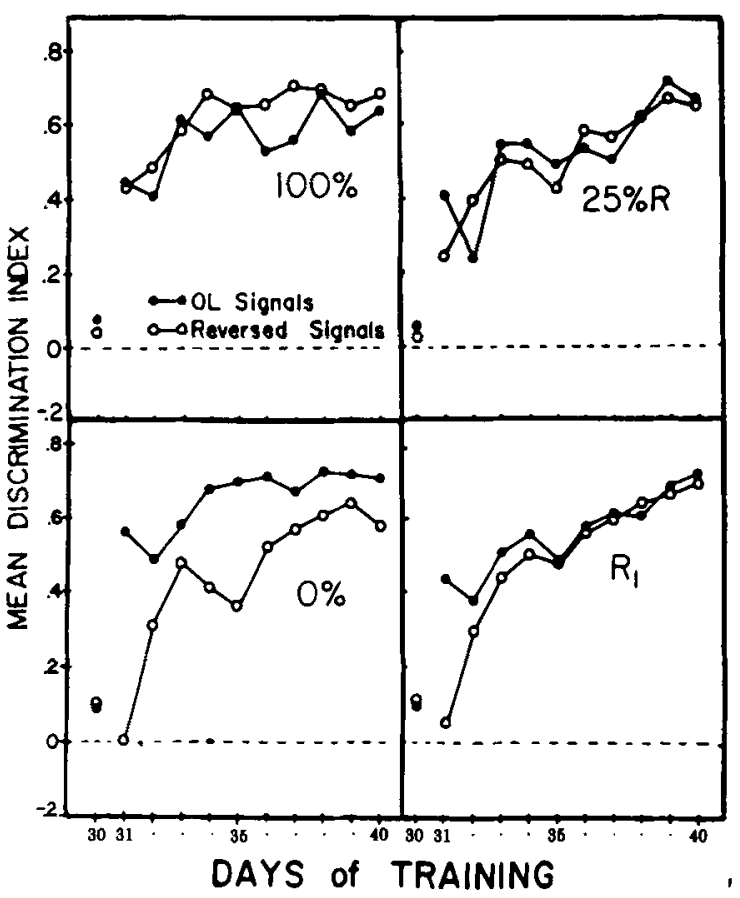

Figure 5. The reversal testing results for halves with their $\mathrm{OL}$ signals retained and the halves with their OL signal shock-pairing revensed. The OL and reversed half performances for the $0 \%$, $100 \%, 25 \% R$, and $R_{1}$ groups, along with performances on the day prior to reversal testing for each half, are shown.

crimination with training $[F s(4,40)=9.45$ and 7.29 , respectively, ps $<.01$ ].

In the $0 \%$ group, the OL half relearned the $S+1$ $\mathrm{S}-$ discrimination more quickly than the reversal half $[F(1,10)=8.94, p<.02]$. Both halves reacquired the discrimination with training $[F(4,40)=5.21$, $\mathrm{p}<.01]$ and, as would be expected from the figure, halves and training interacted $[F(4,40)=3.15, p<.05]$.

For the $R_{1}$ extinction group, the $O L$ and reversal halves were found not to differ, but a significant training $\times$ halves interaction was observed $[F(4,40)=$ $3.67, \mathrm{p}<.02]$. In addition, significant improvement in discrimination with training was observed $[F(4,40)$ $=9.60, p<.01]$. A comparison of the OL and reversal halves of the $R_{1}$ group on Day 31 showed that the OL half discriminated better than the reversal half $[t(10)=3.19, p<.01]$, but only on the 1 st day of reversal testing.

An analysis of the TwR data showed no difference between halves of extinction groups or between groups, no change with training during reversal, and no interactions. Animals continued to respond on about $90 \%$ of all trials, regardless of their grouping.

\section{Discussion}

Considering only the TwR measure, these data constitute a replication of the extinction results reported for goldfish earlier by Wallace and Scobie (1977). The comparison is even more striking when one considers that the $R_{1}$ extinction group had the 
only extinction condition that retained a responsedependent shock-omission contingency, and was thus the logical equivalent of Wallace and Scobie's continued avoidance control. The present data show the same relative relationships (i.e., $R_{1}>100 \%>25 \%$ $>0 \%$ ), including the apparently anomalous TwR performance of the $100 \%$ shock group, as those reported by Wallace and Scobie (1977).

Like Wallace and Scobie, we think that the $100 \%$ shock group maintained its high TwR rate because goldfish do not freeze in situations involving unavoidable shock. In fact, as discussed earlier, it is precisely this behavioral characteristic that allows goldfish to learn to discriminatively respond to signals paired with shock and shock omission. By continuing to respond, they offer the experimenter the opportunity to differentially reinforce signal-specific responses, which eventually results in the acquisition of discriminative responses. Furthermore, and more importantly, the acquisition of signal-specific discriminated responses makes possible the application of another performance indicant-the discrimination index (DI).

Comparing the results of extinction procedures, as depicted in Figures 3 and 4, clearly shows that the TwR and DI measures paint quite different pictures of extinction in goldfish. This is especially true for the $100 \%$ shock group, which maintains TwR performance (Figure 3 ) but shows a substantial drop in discrimination performance on the DI measure (Figure 4). Maintenance of TwR performance is precisely what Wallace and Scobie (1977) observed for their Pavlovian (100\% shock) extinction group, but, without the additional DI discrimination measure, they were forced to conclude that the TwR maintenance was some sort of anomaly, considering the cessation of response typical of most standard laboratory animals trained with $100 \%$ shock in extinction.

However, Bolles (1970) has pointed out that a rat faced with unavoidable shock reverts to its SSDR of freezing. The results for goldfish are anomalous only if one assumes that they possess the same SSDRs as rats, and the evidence points to the contrary. The goldfish's SSDR to procedures involving unavoidable shock is not to freeze but, rather, to become more active (Zerbolio, 1981). In fact, it will be recalled that this pattern of responding is the very basis of the goldfish's ability to learn to respond discriminatively to signals differentially paired with shock and shock omission, since it provides the experimenter with the opportunity to reinforce responses differentially.

While this interpretation explains the seemingly anomalous behavior of the $100 \%$ shock group on the TwR measure, it does so by challenging the adequacy of the TwR measure as a measure of extinction, or, by implication, of learning as well. This challenge is not new. It was clearly anticipated by Steiner (1971) and has been elaborated by further work (Zerbolio, 1981). Based on these and previous data, it seems reasonable to conclude that the TwR measure in goldfish is the result of many things, including the goldfish's learning to respond, its SSDRs to shock, and perhaps the habituation pattern shown by this animal to the shuttlebox situation itself (Zerbolio, 1981). Thus, at best, the TwR measure is confounded in that the effect of learning per se cannot be extricated from the potential effects of other nonlearning behavioral characteristics that have been shown to produce an increase in the TwR response. Given that the TwR measure is confounded for the $100 \%$ shock group, its use as a learning measure for the remaining extinction groups is at best questionable. Fortunately, the linear presentation procedure provides another measure of learning - the DI measure.

Parts of the DI extinction data are consistent with the expectancy position (Bolles, 1978; Davenport \& Olson, 1968). The $100 \%$ shock and $25 \% \mathrm{R}$ groups both have their OL $\mathrm{S}+$ and $\mathrm{S}$ - signals paired nondifferentially with shock, albeit on different schedules $(25 \%$ R vs. $100 \%)$. This would be expected to disrupt the $\mathrm{OL}$ expectancies, especially for the $\mathrm{S}+$ signal, and to produce fairly rapid extinction of the $\mathrm{S}+$ preference. The data are consistent with this interpretation.

The $R_{1}$ and $0 \%$ groups also have their OL $S+$ and $\mathrm{S}$ - signals paired nondifferentially in extinction, but for these groups both $\mathrm{S}+$ and $\mathrm{S}$ - are paired with shock omission. It would be expected that these groups would behave similarly on the DI measure. Furthermore, if pairing both OL signals with shock omission were just as disruptive as pairing both with shock, the $R_{1}$ and $0 \%$ groups should demonstrate a DI in extinction that was comparable to that of the $100 \%$ and $25 \% \mathrm{R}$ groups. This was not the case. While the $0 \%$ group's performance on the DI was comparable to that of the $100 \%$ and $25 \% \mathrm{R}$ groups, the $\mathbf{R}_{1}$ group's was significantly higher.

One possible explanation for the DI difference between the $R_{1}$ and $0 \%$ groups is their marked difference in TwR. This difference in itself is not surprising, because the $R_{1}$ group retains a response-dependent shock-omission contingency in extinction and, like the avoidance control group in a single stimulus procedure, its TwR rate remains high. However, since the DI may be negatively biased for animals which respond on only a few trials, it may underestimate the discrimination performance of the $0 \%$ group. If so, the appropriate $\mathrm{S}+/ \mathrm{S}-$ discrimination measure might be the proportion of TwR in which $S+$ prevails at $T_{10}$. This measure was calculated for all animals. Its plot and analysis were indistinguishable from those of the DI measure, which ruled out the possibility that TwR biased the DI measure.

Another possible explanation of the difference between the $R_{1}$ and $0 \%$ groups is simple generalization 
decrement. It is clear that the $\mathbf{R}_{1}$ extinction procedure, with its shock-omission contingency associated with the TS signal, is closer to the OL procedure than is any other extinction condition. It is equally clear that the $0 \%$ extinction procedure, with shock eliminated entirely, represents the greatest difference from OL. However, while this explanation might account for the differences in extinction between the $0 \%$ and $R_{1}$ groups, it cannot account for the similarity in the extinction results for the $0 \%, 100 \%$, and $25 \% \mathrm{R}$ groups.

In order to relate the extinction treatment and behavior of all four groups, a third extinction measure, based on the reversal learning transfer procedure, is necessary. If the $\mathrm{OL} S+/ \mathrm{S}-$ discrimination had been truly extinguished by the extinction procedure, the half of each group that had its $\mathrm{S}+$ and $\mathrm{S}-$ signals shock-paired as in OL and the half that had its OL signal-shock pairing reversed in the transfer phase would be expected to reacquire the $\mathrm{S}+/ \mathrm{S}-$ discrimination at the same speed. It is clear that, in terms of reversal test interference, the $100 \%$ and $25 \% \mathrm{R}$ groups lost the OL discrimination under extinction training, so that their respective OL and reversed halves reacquired the $\mathrm{S}+/ \mathrm{S}-$ discrimination at equivalent speeds. This finding is consistent with the apparent extinction on the DI measure for these groups during the extinction training phase.

In contrast, neither the $R_{1}$ group nor the $0 \%$ group

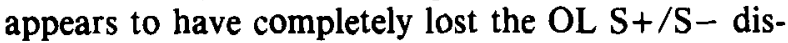
crimination, since both of their reversal group halves learned the $\mathrm{S}+/ \mathrm{S}-$ discrimination more slowly than the respective OL signal halves. Obviously, some amount of the $\mathrm{OL} \mathrm{S}+/ \mathrm{S}-$ discrimination survived extinction training and interfered with reacquisition in the reversal halves of the $0 \%$ and $R_{1}$ extinction groups. Directly comparing the $R_{1}$ and $0 \%$ performances does not shed any further light because, with the exception of the differences already noted, no differences were found. What is clear is that the $\mathrm{OL}$ $\mathrm{S}+/ \mathrm{S}-$ discrimination had not completely extinguished for either the $0 \%$ or the $R_{1}$ group during extinction.

This finding agrees with our original expectation that the $R_{1}$ and $0 \%$ groups would be equal on a discrimination measure following extinction because both would have had the same nondifferential pairing of $S+$ and $S-$ signals during extinction with both $\mathrm{S}+$ and $\mathrm{S}-$ paired with shock omission. However, this suggests an even more interesting implication. In extinction, which expectancy, the $\mathrm{S}+$ or $\mathrm{S}-$, is the more important? For the $100 \%$ and $25 \% \mathrm{R}$ groups, pairing both OL signals with shock can only disrupt the $S+$ expectancy. However, for the $R_{1}$ and $0 \%$ shock groups, pairing both OL signals with shock omission can only disrupt the $\mathrm{S}$ - expectancy. Obviously, disrupting the $\mathrm{S}+$ expectancy is more important than disrupting the $S-$ expectancy, since only the groups with their $S+$ signals disrupted via pairing with shock (100\% and $25 \% \mathrm{R}$ groups) showed extinction.

This does not mean that the $100 \%$ and $R_{1}$ groups did not extinguish. It is clear that some extinction did occur, because the DI measure was not driven below chance performance levels for the reversal halves, as was seen for the reversal group in Experiment 1 and reported earlier by Zerbolio (1981). However, the difference in the amount of extinction for the $0 \%$ and $R_{1}$ groups compared with the $100 \%$ and $25 \% \mathrm{R}$ groups illustrates the basic asymmetry between extinction procedures which disrupt the $\mathrm{S}+1$ shock-omission expectancy vs. those that disrupt the $\mathrm{S}-/$ shock expectancy. Obviously, the traditional procedure of turning off the shocker can never disrupt the $\mathrm{S}+/$ shock-omission expectancy, and, as a result, extinction would be necessarily slower than if the $S+$ was paired on all $(100 \%)$ trials or a random subset $(25 \% \mathrm{R})$ of trials (Davenport \& Olson, 1968).

As an interesting aside, the fact that the $0 \%$ and $\mathbf{R}_{1}$ groups do at least partially extinguish also implies that pairing the $S-$ signal with shock omission does produce extinction, albeit less effectively than pairing the $\mathrm{S}+$ signal with shock.

A further, and perhaps even more fundamentally important, implication can be drawn from these data. Consider the discrepancy between the DI measure for the $0 \%$ group during extinction (Figure 4) and the reversal learning data (Figure 5). During extinction, the $0 \%$ group appeared to lose the OL $S+1$ $\mathrm{S}-$ discrimination. But, when the reversal test was made, it was clear that the $0 \%$ group had retained at least some of its original $\mathrm{S}+/ \mathrm{S}-$ discrimination despite the indications of the DI measure during extinction training. This suggests that, during extinction, although the $0 \%$ group in fact still retained the $\mathrm{S}+/ \mathrm{S}-$ discrimination, it was not detected on the DI measure.

One could argue that the $\mathrm{S}+/ \mathrm{S}-$ discrimination had not extinguished but, rather, had become "latent" in the sense that, while the discrimination still existed, it was not behaviorally manifested until the situation that made the discrimination information relevant (reversal testing) occurred. If this interpretation is correct, it implies that goldfish can appear to lose a response when, in fact, loss has not occurred. All that is required to observe the response is to arrange the proper situation, and the "latent" response will occur again. Thus, it may be possible for a specific response to become "latent" or not measurable, yet still be present. This implication lends credibility to the notion that a fear response, although not detectable behaviorally, could still be present and therefore might still be capable of reinforcing avoidance behavior. If this interpretation is correct, it breathes new life into positions that as- 
sume that fear in an asymptotically avoiding animal can be a central state and, as such, may not be behaviorally measurable (Rescorla \& Solomon, 1967).

\section{GENERAL DISCUSSION}

The results of the present experiments show that the linear presentation procedure, with its additional $\mathrm{S}+/ \mathrm{S}-$ discrimination learning performance measure and its transfer design capability, provides substantially more information about the processes involved in discrimination learning, avoidance learning, and avoidance extinction than do procedures employing only one behavioral measure.

The use of such discrimination learning procedures, with shock and shock-omission reinforcers, may be restricted to animals, like the goldfish, that do not freeze in situations involving uncontrollable shock. But even so, the correspondence between the present results, with goldfish, and the learning literature based on other animals is quite encouraging.

The discrimination acquired is quite consistent with that acquired with food/food-omission procedures in that, as the signal-replacement transfer results of Experiment 1 show, the slightly more important signal is the one paired with the less preferred event-shock. However, this discriminated response is also an avoidance response. When viewed as such, the results have additional implications. In acquisition, the goldfish learns the motivational properties of both the shock-paired and shock-omission-paired signals. If either is retained in transfer, the goldfish maintains its discriminated performance and continues to avoid.

The ability of the goldfish to use the S+ signal to maintain its discriminated avoidance performance at least partially answers Mowrer's (1947) question, "How can a shock which is not experienced, i.e., which is avoided, be said to provide either a source of motivation or of satisfaction?"' In Experiment 1, goldfish used a signal paired with "nonexperienced shock" as the controlling signal in transfer, and it seems reasonable to assume that the $\mathrm{S}+$ signal gained positive motivational value by virtue of its being paired with shock omission. Thus, animals (goldfish) can and do learn to "avoid," not just escape from signals paired with shock.

The data from Experiment 1 also cast light on another issue. Moot (in Bolles, 1978), Morris (1974, 1975), and Weisman and Litner (1969) all showed that if a safety signal is established first via a preliminary classical pairing procedure, the signal can then affect the acquisition and maintenance of an avoidance response. Bolles (1978) argued that such studies, in which the functional properties of a "safety signal" or of a signal paired with shock omission are established outside the avoidance learning situation, do not prove that such a signal or learning mechanism is operative in the avoidance learning situation itself. Experiment 1 shows that the functional properties of the $S+$ signal were learned during the normal course of avoidance acquisition.

The data from Experiment 2 reflect the use of additional performance measures and the reversal transfer design and provide a deeper understanding of the effect of extinction procedures on goldfish. The first finding is that the TwR measure is probably confounded by the goldfish's SSDR to unavoidable shock and therefore is an inappropriate measure of learning.

Once the inadequacy of the TwR measure is recognized, the necessity for an unconfounded performance measure is clearly apparent. The discrimination learning measure, DI, fulfills this requirement, since it provides an index of the goldfish's learning independent of its response rate. This can be seen in Experiment 2's extinction phase, in which the $100 \%$ shock group dropped on the DI measure and not on the TwR measure, and in the transfer testing phase, in which all groups, reversal and OL, performed equivalently on the TwR measure but quite differently on the DI measure.

Use of the DI measure and the reversal test transfer procedure makes it clear that extinction procedures designed to disrupt the goldfish's $\mathrm{S}+$ /shockomission expectancies are much more effective in extinguishing the $\mathrm{S}+/ \mathrm{S}-$ discriminated response than extinction procedures which merely disrupt the $\mathrm{S}-/$ shock expectancies.

The asymmetry between the extinction effects generated by procedures which disrupt the $S+/$ shockomission expectancy and those which disrupt the $\mathrm{S}-$ /shock expectancy confirms the positions taken by theorists (Bolles, Moot, \& Grossen, 1971; Davenport \& Olson, 1968; Mackintosh, 1974) who have argued that the high resistance to extinction attributed to avoidance responses is a function of the experimental extinction procedure itself and not inherent in avoidance learning per se.

The last finding of interest is the contrast between the DI measure during extinction and the results of the reversal transfer procedure for the $0 \%$ shock and $\mathbf{R}_{1}$ extinction groups. These data show that the failure to find an expected result on one behavioral measure does not necessarily mean that the effect would not be detectable by another measure. The problem is to devise a measure sensitive to the effect. There are both positive and negative implications of this finding. On the one hand, it suggests that it is possible to detect an effect with the proper measurement technique, while, on the other hand, it appears to rule out negative findings as evidence for no effect. This last implication, especially, is potentially very problematic. 
However, the main finding of the present work is that a discrimination learning procedure, when applicable, can provide substantially more information about shock-motivated discrimination, or truly discriminated avoidance learning, than can traditional single-stimulus techniques which only nominally represent discrimination.

The applicability of such a design is dependent, to a large extent, upon the response patterns of the animal studied. The goldfish's tendency to increasingly respond in unavoidable shock situations makes it an ideal animal to use with such procedures. Until other animals with the same SSDR to shock situations are found, the goldfish represents the most appropriate and interesting animal to use to study truly discriminated avoidance learning.

\section{REFERENCES}

Bolles, R. C. Species-specific defense reactions and avoidance learning. Psychological Review, 1970, 77, 32-48.

Bolles, R. C. The role of stimulus learning in defensive behavior. In S. H. Hulse, H. Fowler, \& W. K. Honig (Eds.), Cognitive processes in animal behavior. Hillsdale, N.J: Erlbaum, 1978.

Bolles, R. C., Moot, S. A., \& Grossen, N. E. The extinction of shuttlebox avoidance. Learning and Motivation, 1971, 2, 324-333.

Davenpont, D. E., \& Olson, R. D. A reinterpretation of extinction in discriminated avoidance. Psychonomic Science, 1968, 13, 5-6.

Derdzinski, D., \& Warren, J. M. Perimeter, complexity, and form discrimination learning by cats. Journal of Comparative and Physiological Psychology, 1969, 68, 407-411.

Hohner, J. L., Longo, N., \& Bitterman, M. E. A shuttlebox for fish and a control circuit of general applicability. American Journal of Psychology, 1961, 74, 114-120.

JACOBS, B. Repeated acquisition and extinction of an instrumental avoidance response. Journal of Comparative and Physiological Psychology, 1963, 56, 1017-1021.

Jenkins, H. M. Generalization gradients and the concepts of inhibition. In D. I. Mostofsky (Ed.), Stimulus generalization. Stanford: Stanford University Press, 1965.

Mackintosh, N. J. The psychology of animal learning. London: Academic Press, 1974.

Mander, J. M. Overtraining and the use of positive and negative stimuli in reversal and transfer. Journal of Comparative and Physiological Psychology, 1968, 66, 110-115.

Mason, J. R., Stevens, D. A., Wixon, D. R., \& Owens, M. P. Assessment of the relative importance of $S_{+}$and $S-$ in rats using differential training on intercurrent discriminations. Learning and Motivation, 1980, 11, 49-60.
Morris, R. G. M. Pavlovian conditioned inhibition of fear during shuttlebox avoidance behavior. Learning and Motivation, 1974, $5,424-447$.

Morris, R. G. M. Preconditioning of reinforcing properties to an exteroceptive feedback stimulus. Learning and Motivation, 1975, 6, 289-298.

Mowren, $\mathbf{O}$. H. On the dual nature of learning-A reinterpretation of "conditioning" and "problem solving." Harvard Educational Review, 1947, 17, 102-148.

Rescorla, R. A., \& Solomon, R. L. Two-process learning theory: Relationship between Pavlovian conditioning and instrumental learning. Psychological Review, 1967, 74, 151-182.

Steiner, G. Stimulus control of avoidance learning in fish. Journal of Comparative and Physiological Psychology, 1971, $74,52-58$.

Stevens, D. A., Mason, J. R., \& Wixon, D. R. Assessment of the relative importance of $S+$ and $S-$ in rats, using intercurrent simultaneous and successive discriminations. Bulletin of the Psychonomic Society, 1981, 17, 200-202.

Stevens, D. A., \& Fechter, L. D. Relative strengths of approach and avoidance tendencies in discrimination learning of rats trained under two types of reinforcement. Journal of Experimental Psychology, 1968, 76, 489-491.

Wallace, J., \& Scobie, S. R. Avoidance extinction in goldfish. Learning and Motivation, 1977, 8, 18-38.

Weisman, R. G., \& Litner, J. S. Positive conditioned reinforcement of Sidman avoidance behavior in rats. Journal of Comparative and Physiological Psychology, 1969, 68, 597-603.

Woodard, W. T., \& BitTerman, M. E. Pavlovian analysis of avoidance conditioning in the goldfish (Carassius auratus). Journal of Comparative and Physiological Psychology, 1973, 82, 123-129.

Zerbolio, D. J., JR. Discriminated avoidance learning and reversal by goldfish in a shuttlebox using a linear presentation procedure. Animal Learning \& Behavior, 1981, 9, 346-356.

Zerbolio, D. J., \& ReYnierse, J. H. Discriminated learning and reversal in the wheat-turn avoidance situation. Canadian Journal of Psychology, 1967, 21, 185-195.

Zerbolio, D. J., Jr., \& Wickstra, L. L. Goldfish avoidance acquisition: Is the process classical, instrumental, or phototaxis? Bulletin of the Psychonomic Society, 1978, 11, 321-323. (a)

Zenbolio, D. J., JR., \& Wickstra, L. L. Does elimination of a negative phototaxis eliminate CAR acquisition in goldfish? Bulletin of the Psychonomic Society, 1978, 11, 324-326. (b)

ZERBolio, D. J., JR., \& Wickstra, L. L. Instrumentally based conditioned avoidance response acquisition in goldfish in a simultaneous presentation task. Bulletin of the Psychonomic Society, 1979, 13, 311-313.

Zerbolio, D. J., JR., \& Wickstra, L. L. Instrumental avoidance acquisition by goldfish in a $Y$-maze using explicit and responsecontingent cues. Animal Learning \& Behavior, 1980, 8, 304-310.

(Manuscript received September 30, 1981; revision accepted for publication March 9, 1982.) 